\title{
Phytochemical composition and in vitro antioxidant activities of Citrus sinensis peel extracts
}

\author{
Sok Sian Liew ${ }^{1}$, Wan Yong Ho ${ }^{\text {Corresp., }}{ }^{1}$, Swee Keong Yeap ${ }^{2}$, Shaiful Adzni Bin Sharifudin ${ }^{1,3}$ \\ ${ }^{1}$ Department of Biomedical Sciences, The University of Nottingham Malaysia Campus, Semenyih, Selangor, Malaysia \\ 2 China-ASEAN College of Marine Sciences, Xiamen University Malaysia, Sepang, Selangor, Malaysia \\ 3 Biotechnology Research Centre, Malaysian Agricultural Research and Development Institute (MARDI), Serdang, Selangor, Malaysia \\ Corresponding Author: Wan Yong Ho \\ Email address: WanYong.Ho@nottingham.edu.my
}

Background. Citrus sinensis peels are usually discarded as wastes, however, they are rich sources of Vitamin C, fibre, and many nutrients, including phenolics and flavonoids which are also good antioxidant agents. This study aimed to examine phytochemical composition and antioxidant capabilities of $C$. sinensis peel extracted conventionally with different methanol/water, ethanol/water, and acetone/water solvents. Methods. $C$. sinensis peel were subjected to extraction with $100 \%, 70 \%$ and $50 \%$ of methanol, ethanol, and acetone respectively, as well as hot water extraction. Antioxidant activities of the peel extracts were examined via the 2,2-diphenylpicrylhydrazyl (DPPH) free radical scavenging activity, ferric reducing antioxidant power (FRAP) assay, and oxygen radical absorbance capacity (ORAC) assay. Total phenolic content and total flavonoid content of the extracts were then measured via Folin-Ciocalteau and aluminium chloride colorimetric method respectively. Phenolic acid and organic acid composition of the peel extracts were further determined via High Performance Liquid Chromatography (HPLC) while flavonoid content was identified via Ultra Performance Liquid Chromatography (UPLC). Results. DPPH radical scavenging activity of $C$. sinensis peel extracts varied from 8.35 to $18.20 \mathrm{mg}$ TE/g, FRAP ranged from 95.00 to $296.61 \mathrm{mmol} \mathrm{Fe}(\mathrm{II}) / \mathrm{g}$, while ORAC value ranged from 0.31 to $0.92 \mathrm{~mol} \mathrm{TE} / \mathrm{g}$.

Significant level of association between the assays was observed especially between TPC and FRAP (R-square $=0.95, P<0.0001$ ). TPC of various $C$. sinensis peel extracts ranged from 12.08 to $38.24 \mathrm{mg} \mathrm{GAE} / \mathrm{g}$, with $70 \%$ AEC showing the highest TPC. TFC ranged from 1.90 to $5.51 \mathrm{mg} \mathrm{CE} / \mathrm{g}$. Extraction yield ranged from 0.33 to $0.52 \mathrm{~g} / \mathrm{g} \mathrm{DW}$ and tended to increase with increasing water concentration in the solvent. In phytochemical investigation, five phenolic acids were identified using HPLC, including gallic acid, protocatechuic acid, 4hydroxybenzoic acid, caffeic acid and ferulic acid. A total of five organic acids including lactic acid, citric acid, L-mallic acid, kojic acid and ascorbic acid were quantified via HPLC. Besides that, concentrations of six flavonoids including catechin, epigallocatechin, vitexin, 
rutin, luteolin and apigenin were determined via UPLC. Discussion and Conclusion Phytochemicals including phenolics and flavonoids in C. sinensis peel extracts exhibited good antioxidant properties. Among the extracts, 70\% AEC with highest TPC and high TFC content showed greatest antioxidant activity in all three assays. Different phenolic acids, organic acids and flavonoids were also identified from the extracts. This study indicated that $C$. sinensis peels contained potential antioxidant compounds which could be exploited as value added products in the food industry. 


\section{Phytochemical composition and in vitro antioxidant activities of Citrus sinensis peel extracts}

Sok Sian Liew ${ }^{1}$, Wan Yong Ho${ }^{1}$, Swee Keong Yeap ${ }^{2}$, Shaiful Adzni Bin Sharifudin ${ }^{1,3}$

${ }^{1}$ Department of Biomedical Sciences, University of Nottingham Malaysia Campus, Semenyih, Selangor, Malaysia.

${ }^{2}$ China-ASEAN College of Marine Sciences, Xiamen University Malaysia, Sepang, Selangor, Malaysia

${ }^{3}$ Biotechnology Research Centre, Malaysian Agricultural Research and Development Institute (MARDI), Serdang, Selangor, Malaysia

Corresponding Author:

Wan Yong Ho

Department of Biomedical Sciences, University of Nottingham Malaysia Campus, Semenyih, Selangor, 43500, Malaysia.

Email address: WanYong.Ho@nottingham.edu.my 
1 Abstract

2 Background. Citrus sinensis peels are usually discarded as wastes, however, they are rich

3 sources of Vitamin C, fibre, and many nutrients, including phenolics and flavonoids which are

4 also good antioxidant agents. This study aimed to examine phytochemical composition and

5 antioxidant capabilities of $C$. sinensis peel extracted conventionally with different

6 methanol/water, ethanol/water, and acetone/water solvents.

7 Methods. C. sinensis peel were subjected to extraction with $100 \%, 70 \%$ and $50 \%$ of methanol, 8 ethanol, and acetone respectively, as well as hot water extraction. Antioxidant activities of the 9 peel extracts were examined via the 2,2-diphenylpicrylhydrazyl (DPPH) free radical scavenging 10 activity, ferric reducing antioxidant power (FRAP) assay, and oxygen radical absorbance 11 capacity (ORAC) assay. Total phenolic content and total flavonoid content of the extracts were then measured via Folin-Ciocalteau and aluminium chloride colorimetric method respectively. Phenolic acid and organic acid composition of the peel extracts were further determined via High Performance Liquid Chromatography (HPLC) while flavonoid content was identified via Ultra Performance Liquid Chromatography (UPLC).

Results. DPPH radical scavenging activity of $C$. sinensis peel extracts varied from 8.35 to 18.20 mg TE/g, FRAP ranged from 95.00 to $296.61 \mathrm{mmol} \mathrm{Fe(II)/g,} \mathrm{while} \mathrm{ORAC} \mathrm{value} \mathrm{ranged} \mathrm{from}$ 0.31 to $0.92 \mathrm{~mol} \mathrm{TE} / \mathrm{g}$. Significant level of association between the assays was observed especially between TPC and FRAP (R-square $=0.95, \mathrm{P}<0.0001)$. TPC of various $C$. sinensis peel extracts ranged from 12.08 to $38.24 \mathrm{mg} \mathrm{GAE} / \mathrm{g}$, with 70\% AEC showing the highest TPC. TFC ranged from 1.90 to $5.51 \mathrm{mg} \mathrm{CE} / \mathrm{g}$. Extraction yield ranged from 0.33 to $0.52 \mathrm{~g} / \mathrm{g} \mathrm{DW}$ and tended to increase with increasing water concentration in the solvent. In phytochemical investigation, five phenolic acids were identified using HPLC, including gallic acid, protocatechuic acid, 4hydroxybenzoic acid, caffeic acid and ferulic acid. A total of five organic acids including lactic acid, citric acid, L-mallic acid, kojic acid and ascorbic acid were quantified via HPLC. Besides that, concentrations of six flavonoids including catechin, epigallocatechin, vitexin, rutin, luteolin and apigenin were determined via UPLC.

Discussion and Conclusion Phytochemicals including phenolics and flavonoids in C. sinensis peel extracts exhibited good antioxidant properties. Among the extracts, 70\% AEC with highest 
TPC and high TFC content showed greatest antioxidant activity in all three assays. Different phenolic acids, organic acids and flavonoids were also identified from the extracts. This study indicated that $\mathrm{C}$. sinensis peels contained potential antioxidant compounds which could be exploited as value added products in the food industry.

\section{Introduction}

Citrus sinensis, also known as sweet orange, is consumed not only as a fruit but also as medicinal herb in some nations. It belongs to the Rutaceae family and is widely distributed in the tropical and subtropical regions. Annual worldwide citrus fruit production now stands at over 110 million tons and orange has become the most commonly grown fruit in the world (Blauer, 2016). Out of the forecasted global production of 24 million tons of oranges in year 2016/17, it is estimated that around $8.3 \%$ (2 million tons) of these will be used for orange juice production (Foreign Agricultural Service/USDA, 2017). However, orange peels accounts for around 44\% of the fruit body ( $\mathrm{Li}$ et al. 2006) and thus will produce a huge mass of by-products. These orange peels are usually discarded as wastes leading to serious disposal problem that may be detrimental to the environment.

Considering the huge quantity of these "wastes" that are produced in the food supply chain, orange peels offers a huge potential to be exploited as value-added product, including for the recovery of natural antioxidants, pectin, enzymes or for the production of ethanol, organic acids, essential oils and prebiotics single cell protein (Mamma and Christakopoulos, 2014). In addition, C. sinensis peel is a rich source of Vitamin C, fibre, and many nutrients, including phenolics and flavonoids. It is subdivided into two main parts, epicarp and mesocarp. Epicarp is the coloured peripheral surface, largely made of parenchymatous cells and cuticle. It is covered with an epidermis of epicuticular wax with many small aromatic oil glands giving its particular smell. Mesocarp is the soft whitish middle layer lying beneath epicarp. It is made up of tubular-like cells connecting together to create the tissue mass compressed into the intercellular area (FavelaHernández et al., 2016). C. sinensis peel has been used as a traditional medicine in certain parts of the world for relieving stomach discomfort, skin inflammation, ringworm infections, aiding in neuroprotection, and improving heart health (Li, Lo and Ho. 2006; Ghasemi et al. 2009). 
58 Various potent antioxidants have been found in citrus peels and showed antioxidant effect 59 including free radical scavenging and metal chelation activities. It is much encouraging to 60 explore the active phytochemicals in C. sinensis peel as reactive oxygen species is playing a 61 main role in many diseases such as cancer, cardiovascular dysfunction, neurodegenerative 62 diseases, and process of ageing (Rafiq et al., 2016). A recent study on identification of 4'geranyloxyferulic (GOFA) among citrus peel extracts revealed that $C$. sinensis has the richest content of GOFA which previously showed neuroprotective and dietary feeding colon cancer chemopreventive effects in rats (Genovese and Epifano, 2012; Genovese et al., 2014). A group of flavonoids, polymethoxyflavones (PMFs), which is found abundantly and almost only from the citrus peels, have been given great attention because of their wide range of properties. Many in vitro experiments elucidated anticancer actions by PMFs such as antiproliferation, enzyme inhibition and cancer cell growth inhibition (Qiu et al., 2011; Onda et al., 2013; Rawson, Ho and $\mathrm{Li}, 2014)$.

Extraction is the key step for analysis and exploitation of plant bioactive compounds. An ideal extraction method should be quantitative, non-destructive, and time effective. Due to lower toxicity and ease of access to water, traditional method of using medicinal plants was boiled in water and consumed the extracts as additives in food or consumed directly as functional foods but the effectiveness of consuming boiled water extract was in doubt (Wong et al., 2006). Conventional solvent extraction (CSE) is used widely for the recovery of bioactive compounds due to its simplicity. Despite its simplicity, disadvantages such as long extraction time, large consumption of solvents, exposure to flammable and hazardous liquid organic solvents, conventional method of extraction encourages use of more advanced technique. Some of the non-conventional extraction methods were suggested, however CSE was quite comparable to other extraction method in the TPC and antioxidant activity of extracts as shown in study by Nayak et al., (2015). Phenolics or antioxidant content is greatly affected by properties of the extracting solvents. Some common solvent used in extraction of phenolics are methanol, ethanol, propanol, acetone and ethyl acetate (Spigno, Tramelli and De Faveri, 2007). Phenolic compounds dissolve better in solvent with higher polarity such as methanol. It is important to note that some organic solvents are identified as toxic such as methanol, therefore, ethanol as a 
87 food-grade solvent is preferred to be used for the extraction of phenolic compounds from various

88 citrus peels (Li, Smith and Hossain, 2006).

89

90

Due to their low cost and high availability in the world, $C$. sinensis peels and its phytochemical compounds could serve as a cheap and yet nutritional dietary supplement or even a potential therapeutic agent. However, its true properties should be proven with more reliable and systematic study to understand the role of $C$. sinensis in performing these beneficial health actions. It was hypothesized that conventional extraction with the solvents including methanol, ethanol, and acetone can isolate the useful bioactive compounds which will exert high antioxidant activities. Therefore, this study aims to examine antioxidant capabilities of $C$. sinensis peel extracts and the correlation to their phytochemical content.

\section{Materials \& Methods}

\section{Extraction and preparation of samples}

. sinensis peels are by-products collected from an orange juice manufacturer. The peels composed of flavedo and albedo were then washed and fully dried in an oven at $60^{\circ} \mathrm{C}$ for 72 hours. The dried peels were ground to powder with particle size ranging of $0.5 \mathrm{~mm}$ to $0.1 \mathrm{~mm}$ using mortar and pestle and were extracted using 100/0, 70/30, 50/50\% (v/v) of methanol/water, ethanol/water, and acetone/water solvents respectively for 72 hours with a mass to volume ratio of 1:25 (g/ml). The extracts were then filtered through Whatman No.1 filter paper and collected into glass bottles. The whole process of extraction and filtration was repeated twice followed by evaporation of the collected extracts to dryness using a rotary evaporator at $37^{\circ} \mathrm{C}$. The extracts were re-dissolved in dimethyl sulphoxide (DMSO) to a concentration of $100 \% \mathrm{mg} / \mathrm{mL}$ and kept at $4{ }^{\circ} \mathrm{C}$ until use. Extraction yield was expressed in $\mathrm{g}$ of extract per $\mathrm{g}$ of dry weight $(\mathrm{g} / \mathrm{g} \mathrm{DW})$. For water extract, $4 \mathrm{~g}$ of dried $C$. sinensis peel powder was boiled with distilled water for 1 hour before filtering it through Whatman No.1 filter paper. The whole process of extraction and collection of extract was repeated again followed by evaporating the collected extract to $40 \mathrm{~mL}$ left (Concentration: $4 \mathrm{~g} / 40 \mathrm{~mL}=100 \mathrm{mg} / \mathrm{mL}$ ) using a boiling water bath. Water extract stock was stored in $-20^{\circ} \mathrm{C}$ and thawed before use. Extracts of $C$. sinensis peel by using 100, 70, $50 \mathrm{wt} . \%$ methanol/water and ethanol/water solvents were expressed as 100\% MEC, 70\% MEC, 50\% 
$115 \mathrm{MEC}, 100 \% \mathrm{EEC}, 70 \% \mathrm{EEC}$, and 50\% EEC, respectively. Extracts of $C$. sinensis peel by using

11670 and 50 wt.\% acetone/water solvents were expressed as 70\% AEC, and 50\% AEC

117 respectively. Water extract of $C$. sinensis peel were expressed as WEC.

\section{Determination of 2,2-diphenyl-1-picrylhydrazyl (DPPH) radical scavenging activity}

119 The scavenging activity of the extract against DPPH $\bullet$ radical was measured according to Brand-

120 Williams et al. method (Brand-Williams, Cuvelier and Berset, 1995). $50 \mu \mathrm{L}$ of $2.5 \mathrm{mg} / \mathrm{mL}$

121 extract was added to $150 \mu \mathrm{L}$ of mixture $(0.2 \mathrm{mM} \mathrm{DPPH} \bullet$ and $90 \mathrm{mM}$ Tris-Cl). The total volume

122 was made up to $200 \mu \mathrm{L}$ with methanol. The mixture was incubated in dark for 40 minutes at

$12325^{\circ} \mathrm{C}$ before taking the absorbance readings at $517 \mathrm{~nm}$. DPPH• scavenging radical ability of each

124 sample was expressed as mg of trolox equivalents per $\mathrm{g}$ of sample (mg TE/g).

\section{Determination of ferric reducing antioxidant power (FRAP)}

126 FRAP assay was performed with slight modification (Benzie and Strain, 1996). The FRAP

127 reagent was prepared by mixing TPTZ $(1 \mathrm{mM}), \mathrm{FeCl} 3 \bullet 6 \mathrm{H} 2 \mathrm{O}(2 \mathrm{mM})$, and $300 \mathrm{mM}$ acetate

128 buffer in a ratio of $10: 1: 1$ at $37^{\circ} \mathrm{C} .25 \mu \mathrm{L}$ of $1 \mathrm{mg} / \mathrm{mL}$ extract was mixed with $175 \mu \mathrm{L}$ FRAP

129 reagent. A final volume of $200 \mu \mathrm{L}$ reaction mixture was incubated in dark for 10 minutes at $25^{\circ} \mathrm{C}$

130 before taking the absorbance readings at $590 \mathrm{~nm}$. FeSO4 $7 \mathrm{H} 2 \mathrm{O}$ with different concentrations

$131(100-1000 \mu \mathrm{M})$ was used as standard for construction of calibration curve. FRAP value of each

132 sample was expressed as mmol of $\mathrm{Fe}(\mathrm{II})$ per $\mathrm{g}$ of sample (mmol $\mathrm{Fe}(\mathrm{II}) / \mathrm{g})$.

\section{Determination of oxygen radical absorbance capacity (ORAC)}

134 ORAC assay was carried out according to method of Huang et al. with modifications (Huang et

135 al., 2002). First, $50 \mu \mathrm{L}$ of $100 \mu \mathrm{g} / \mathrm{mL}$ extract was added to $800 \mathrm{nM}$ fluorescein in $75 \mathrm{mM}$

136 phosphate buffer $\mathrm{pH}$ 7.4. The reaction mixture was incubated at $37^{\circ} \mathrm{C}$ for 15 minutes following

137 by addition of $200 \mathrm{mM}$ AAPH (2,2'-azobis-2- methyl-propanimidamide, dihydrochloride)

138 solution to a final volume of $200 \mu \mathrm{L}$. The fluorescence signal was measured using Hitachi F-

1397000 Fluorescence Spectrophotometer in 5 minutes interval over 90 minutes by excitation at 485

$140 \mathrm{~nm}$, emission at $520 \mathrm{~nm}$. Inhibition of loss of fluorescein fluorescence is proportional to the

141 antioxidant effect of the sample. Trolox was used as standard for construction of calibration

142 curve. 
143 The area under the curve (AUC) of each sample was calculated by integrating the relative

144 fluorescence curve. Next, net AUC of the sample was calculated by subtracting the AUC of the 145 blank from the AUC of the sample.

$146 \quad \mathrm{AUC}=1+\mathrm{RFU} 1 / \mathrm{RFU} 0+\mathrm{RFU} 2 / \mathrm{RFU} 0+\ldots . .+\mathrm{RFU} 18 / \mathrm{RFU} 0$

147 where RFU0 = relative fluorescence value of time point zero,

$148 \mathrm{RFUx}=$ relative fluorescence value for the number of reading

149 (eg. RFU5 is relative fluorescence value of fifth reading, which is at minute 25)

150 Net AUC $=$ AUC (sample) - AUC (blank)

151 The regression equation between net AUC and Trolox concentrations was determined and the

152 ORAC value of extracts was expressed as mol trolox equivalents per gram of sample (mol TE/g).

\section{Determination of total phenolic content}

154 TPC of the extracts was determined according to the Folin-Ciocalteu method (Singleton and 155 Rossi, Joseph A., 1965). $250 \mu \mathrm{L}$ of $2 \mathrm{~N}$ Folin-Ciocalteu reagent was mixed with $50 \mu \mathrm{L}$ of 10 $156 \mathrm{mg} / \mathrm{mL}$ extract, following by addition of $750 \mu \mathrm{L}$ of $7 \% \mathrm{w} / \mathrm{v}$ Na2CO3 after 5 minutes. The total 157 volume was made up to $5 \mathrm{~mL}$ with distilled water. The mixture was incubated in dark for 2 hours 158 at $25^{\circ} \mathrm{C}$ before absorbance was measured using a spectrophotometer (The VersaMax ${ }^{\mathrm{TM}}$ 159 Microplate Reader, USA) at $765 \mathrm{~nm}$. TPC results were expressed as mg gallic acid equivalents 160 per g of dry weight (mg GAE/g DW).

\section{Determination of total flavonoid content}

162 TFC of extracts was determined using the aluminium chloride colorimetric assay with slight 163 modification (Zhishen, Mengcheng and Jianming, 1999). $25 \mu \mathrm{L}$ of $10 \mathrm{mg} / \mathrm{mL}$ extract was added with $7.5 \mu \mathrm{L}$ of $\mathrm{NaNO} 2(5 \% \mathrm{w} / \mathrm{v})$, and $7.5 \mu \mathrm{L}$ of $\mathrm{AlCl} 3(10 \% \mathrm{w} / \mathrm{v})$. The mixture was then allowed to stand for 10 minutes at $25^{\circ} \mathrm{C} .50 \mu \mathrm{L}$ of $\mathrm{NaOH}(1 \mathrm{M})$ was added subsequently and the total volume was made up to $250 \mu \mathrm{L}$ with distilled water. The absorbance was measured at $510 \mathrm{~nm}$ using a spectrophotomoter. TFC results were expressed as $\mathrm{mg}$ of catechin equivalents per $\mathrm{g}$ of dry weight (mg CE/g DW).

\section{Quantification of phenolic acids content}


170 Identification and quantification of phenolic acids in the orange peel extracts was performed

171 using a High Performance Liquid Chromatography (HPLC) method using 2956 LC system

172 (Waters, USA). Samples were filtered through $0.22-\mu \mathrm{m}$ pore size membrane filters before

173 injection. Presence of phenolic acids was then determined using a reversed phase XBridge C18

174 column $(4.6 \times 100 \mathrm{~mm}, 3.5 \mu \mathrm{m}$ particle size $)$ and the detector was set at $\Lambda=270 \mathrm{~nm}$, and $\Lambda=306 \mathrm{~nm}$.

175 The separation of phenolic acids was made in gradient condition at $30^{\circ} \mathrm{C}$, using a mobile phase $\mathrm{A}$

176 made of acid water $(0.1 \%$ formic acid) and mobile phase B, methanol (100\%) with the flow rate

177 of $0.7 \mathrm{~mL} / \mathrm{min}$. The gradient elution was performed as follows: $0-10 \mathrm{~min}$, from $95 \%$ to $85 \% \mathrm{~A}$;

$17810-20 \mathrm{~min}$, - from $85 \%$ to $80 \% \mathrm{~A} ; 20-52 \mathrm{~min}$, from 80 to $70 \% \mathrm{~A}$; $52-55 \mathrm{~min}$, maintained at $70 \%$

$179 \mathrm{~A} ; 55$-58 min, from 70 to $50 \% \mathrm{~A}$; 58-63 min, from 50 to $20 \% \mathrm{~A} ; 63-70 \mathrm{~min}$, from 20 to $95 \% \mathrm{~A}$;

180 70-75 min, maintained at 95\% A. Peak identification was made by comparing retention time of

181 known phenolic acids and quantification was performed using calibration curves obtained by

182 injecting known amounts of the pure phenolic acids (Gallic acid, vanillic acid, protocatechuic

183 acid, syringic acid, 4-hydroxybenzoic acid, caffeic acid, o-coumaric acid, ferulic acid, sinapic

184 acid and p-coumaric acid) as the external standards.

185

186

187

188

189

190

191

192

193

194

195

196

197

198

\section{Determination of organic acids content}

HPLC analyses of organic acid content was carried out using 2695 Alliance Separation Module (Waters, USA) equipped with a 2996 diode array detector (Waters, USA). A $10 \mu \mathrm{L}$ aliquot of filtered sample was separated using Synergi Hydro-RP80A column (250 x 4.6 mm, $4 \mu \mathrm{m}$ particle size) (Phenomenex, USA) with temperature controlled at $30^{\circ} \mathrm{C}$. The mobile phase consisted of mobile phase A (20 mM KH2PO4 with adjusted $\mathrm{pH} 2.9)$ and mobile phase B (water) with a flow rate of $0.6 \mathrm{~mL} / \mathrm{min}$. Gradient elution was performed as follows: 0-30 min, maintained at 100\% A; 30-31 min, from $100 \%$ to $0 \% \mathrm{~A} ; 31-45 \mathrm{~min}$, maintained at $0 \% \mathrm{~A} ; 45-46 \mathrm{~min}$, from 0 to $100 \%$ A; 46-55 min, maintained at 100\%. Peak identification was made by comparing retention times and UV spectra at 190, 210 and $254 \mathrm{~nm}$ with authentic organic acids compounds. Quantification was performed using calibration curves obtained by injecting known amounts of pure organic acids (tartaric acid, lactic acid, acetic acid, citric acid, succinic acid, oxalic acid, L-mallic acid, kojic acid and ascorbic acid) as external standards.

\section{Determination of flavonoids content using UPLC}


199 The flavonoid content of the filtered extracts were separated using AcquityTM Ultra

200 Performance Liquid Chromatography (UPLC) system (Waters, USA) with Kinetex C18 100A

201 column $(100 \mathrm{mmx} 2.1 \mathrm{~mm} ; 1.7 \mu \mathrm{m}$ particle size), at a flow rate of $0.4 \mathrm{ml} / \mathrm{min}$ with the temperature

202 controlled at $40^{\circ} \mathrm{C}$ under the UV spectrum of $280,330,360 \mathrm{~nm}$. The gradient elution consists of

203 mobile phase A (water:acetic acid, 97:3) and mobile phase B (methanol). The gradient elution

204 was conducted as follows; 0-1 min, maintained at 100\% A; 1-10 min, from 100 to $40 \% \mathrm{~A} ; 0-12$

$205 \mathrm{~min}$, from 40 to $100 \% \mathrm{~A}$ and then maintained at 100\% A for another $2 \mathrm{~min}$. Quantification was

206 performed using calibration curves obtained by injecting known amounts of flavonoids

207 (Epigallocatechin, vitexin, rutin, quercetin, luteolin, apigenin, tannic acid and ellagic acid) as

208 external standards with known retention time.

\section{Statistical analysis}

210 All experiments were performed in triplicate unless stated otherwise. Statistical analyses of the

211 experimental data were performed with GraphPad prism 6 statistical software (GrapPad

212 Software, USA). Results of the replicates were expressed as mean \pm standard error (SEM). One-

213 way analysis of variance (ANOVA) with Tukey's multiple comparisons test was used to evaluate

214 differences between means in each experiment. Experimental results were further analyzed for

215 Pearson correlation coefficient (R-square) between TPC, TFC and different antioxidant assays. P

216 value of $\leq 0.05$ was taken as statistically significant.

\section{Results}

\section{Antioxidant activity}

Antioxidant activities of the extracts were evaluated via 2,2-diphenyl-1-picrylhydrazyl (DPPH) radical scavenging activity, ferric reducing antioxidant power (FRAP), and oxygen radical absorbance capacity (ORAC). DPPH radical scavenging activity of $\mathrm{C}$. sinensis peel extracts

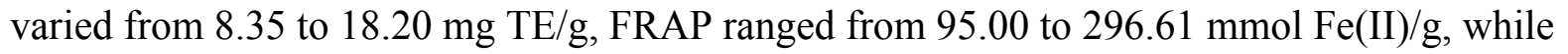
ORAC ranged from 0.31 to $0.92 \mathrm{~mol} \mathrm{TE} / \mathrm{g}$. In all three assays, $70 \%$ AEC showed higher antioxidant activity and WEC showed a much lower antioxidant activity among the extracts 
226 (Table 1). However, DPPH, FRAP and ORAC values of extracts were much lower than the

227 tested positive controls, ascorbic acid and gallic acid. Pearson correlation coefficients (R-square)

228 between TPC, TFC and different antioxidant assays were tabulated in Table 2. Significant level 229 of association between the assays was observed especially between FRAP values and TPC (R230 square $=0.95, \mathrm{P}<0.0001)$ as well as TFC (R-square $=0.93, \mathrm{P}<0.0001)$ across all the extracts.

\section{Extraction yield, total phenolic and total flavonoid content}

233

234

235

236

237

238

239

240

241

242

243

244

245

246

247

248

249

250

251

252

In general, extraction yield of all samples ranged from 0.33 to $0.52 \mathrm{~g} / \mathrm{g} \mathrm{DW}$ and appeared to increase with increasing water concentration in the solvent (Table 3). 100\% AEC was excluded from further study due to its low yield $(<0.005 \mathrm{~g} / \mathrm{g} \mathrm{DW})$ and low solubility (Table 3$)$. TPC of various $C$. sinensis peel extracts ranged from 12.08 to $38.24 \mathrm{mg} \mathrm{GAE} / \mathrm{g}$, with $70 \%$ AEC showing the highest TPC. The other extracts exhibited relatively high TPC too except for $100 \%$ EEC and WEC which showed significantly lower TPC than the other extracts. On the other hand, TFC ranged from 1.90 to $5.51 \mathrm{mg} \mathrm{CE} / \mathrm{g}$. 50\% AEC showed the highest TFC, followed by $70 \% \mathrm{AEC}$. Generally, aqueous acetone extracts contained higher TPC and TFC than the other extracts while 100\% EEC and WEC contained the lowest TPC and TFC among all.

\section{Phytochemical analysis}

Phenolic acids can be divided into derivatives of benzoic acid and derivatives of cinnamic acid and both derivatives were found in our peel extracts. Cinnamic acid derivatives namely ferulic acid and caffeic acid are found in highest abundance while the derivatives of benzoic acid such as gallic acid, protocatechuic acid and 4-hydroxybenzoic acid are present on lower abundance as compared to the former group (Table 4).

Flavonoids are classified into six groups including flavanone, flavonol, flavone, isoflavone, flavan-3-ols, and anthocyanin. The major class of flavonoids in the extracts appear to be the flavan-3-ols (catechin and epigallocatechin), followed by flavanone (luteolin, apigenin and vitexin) while flavonol (rutin) is present at low abundance in the extracts (Table 4). 
253 On the other hand, a few organic acids were identified in the extracts via HPLC (Table 4).

254 Interestingly, 100\% MEC, 100\% EEC and 70\% EEC were shown to contain only lactic acid.

255 Citric acid is the major organic acid in the remaining extracts, followed by lactic acid and L-

256 mallic acid. Kojic acid and ascorbic acid appear to be present in much lower abundance in the 257 extracts.

258

259

260

261

262

263

264

265

266

267

268

269

270

271

272

273

274

275

276

277

278

279

280

\section{Discussion}

In this study, antioxidant activities of $C$. sinensis peel extracts were evaluated and correlated with the important phytochemical content including phenolic acid, flavonoid and organic acid. Antioxidants can deactivate radicals via two main mechanisms, hydrogen atom transfer (HAT) and single electron transfer (SET). In HAT, antioxidant donate hydrogen atoms to stabilise freeradical species to quench them from progressing further in radical reactions while in SET, free radicals are reduced through the donation of an electron from antioxidant compounds (Craft et.al,. 2012). Depending on the structure and properties of the antioxidants present, either HAT or SET may dominate in a given system (Prior, Wu and Schaich, 2005). Therefore, antioxidant capacities of plant extracts greatly depends on extract composition as well as conditions and mechanism of the test used. In order to evaluate antioxidant activity of components in the extract, three antioxidant assays operated on different mechanism were used in our study; FRAP and ORAC assay measures via HAT and SET respectively. DPPH assay determines antioxidant activity via both mechanism (Prior, Wu and Schaich, 2005). In both ethanol and acetone extracts, greatest antioxidant activity was observed in $70 \%$ followed by $50 \%$ and $100 \%$ of both extracts in all three assays (Table 1). This suggests that the water content in the extracts may not correlate proportionally to the antioxidant level but addition of water to the extraction could improve the antioxidant level of the ethanol and acetone extracts. In contrast, antioxidant level of the methanol extracts did not correlate well to water content in the extracts. $100 \%$ MEC showed antioxidant activity that was superior to $70 \%$ and 50\% MEC in both FRAP and ORAC assays but the reverse in DPPH assay. 
281 Antioxidant capacity of phenolics and flavonoids in plants is the main contributor to the specific

282 biological actions in diseases prevention and treatment (Dai and Mumper, 2010). Therefore,

283 bioactive phytochemical components may define the medicinal value of a plant source. From the

284 results, we observed a high correlation between FRAP values and TPC and TFC across all the

285 extracts (Table 2). The higher correlation of TPC and TFC to FRAP values suggest to us that the

286 antioxidant secondary metabolites, in particular phenolics and flavonoids in the peel extracts,

287 may react with free radicals mainly via SET mechanism. Overall, statistically significant

288 correlation between TPC, TFC and the antioxidant assays suggests that the phenolic and

289 flavonoid content contribute to antioxidant activities of the $C$. sinensis extracts.

290 Phenolics or antioxidant content is greatly affected by properties of the extracting solvents

291 (Spigno et al. 2007). Phenolic compounds are generally known to dissolve better in solvents with

292 higher polarity. Polar alcohol type solvent would produce higher yield as compared to other type

293 of solvents. Extraction yield may be increased with addition of water to ethanol, but water

294 content in the solvent would increase concomitant extraction of other compounds, yielding lower

295 concentration of phenols in the extracts (Naczk \& Shahidi 2006). In agreement to the former, we

296 found that the yield of extract increased with increasing water percentage within each solvent

297 extraction group. Although there isn't a specific pattern observed with regards to the effect of

298 addition of water to TPC or TFC content, $30 \%$ of water content in each of the solvent extraction

299 group generally exhibited higher TPC and TFC content than those with $50 \%$ of water content

300 (Table 3). The optimum extraction method, water at boiling temperature was one of the effective

301 solvents for antioxidants extraction giving higher total phenol content (Sousa et al., 2008). In our

302 study, WEC had exerted antioxidant activities and tested to have phenolic and flavonoid content

303 although being the least among the extracts (Table $1 \&$ 3).It was understood that conventional

304 solvent extraction (CSE) is generally being used to extract bioactive components from the plant

305 materials at a small scale level. Our study which used CSE served as a control method to

306 understand the bioactivities of $C$. sinensis peels and a reference method for small scale

307 production or homemade level. However, the challenges to scale up the extraction with this

308 convention method would be long extraction time and large consumption of solvent. Therefore,

309 new and promising non-conventional extraction techniques were introduced for industrial

310 application such as ultrasound-assisted extraction (UAE), molecular distillation, microwave-

311 assisted extraction (MAE), pulsed electric field extraction, and supercritical fluid extraction 
312 (Selvamuthukumaran and Shi, 2017). A recent study comparing CSE, UAE, MAE and

313 supercritical $\mathrm{CO}_{2}$ extraction of Maltease citrus peel showed that MAE was a more effective

314 method in phenols and flavonoids extraction while CSE gave an extract with more antioxidant

315 activity (Boudhrioua, 2016). In another study by Ko, Kwon and Chung (2016), a pilot-scale

316 subcritical water extraction plant was conducted to extract antioxidant flavonoids from dried

317 satsuma mandarin peel (Citrus unshiu Markovich) and the proportion of flavonoids recovered

318 with this extraction pilot plant was $96.3 \%$.

319 Besides important role of extraction solvent, pre-extraction and extraction conditions and

320 methods are equally important in extracting compounds from plant materials. A study by Hegde

321 et al. (2015) recommended peel drying since loss of water content from the peel decreases the

322 bulk of the material for easier handling and storage, lower risk of bacterial growth, as well as

323 more efficient extraction. The study concluded that extraction of sundried peel with acidified

324 aqueous methanol at $90^{\circ} \mathrm{C}$ for 5 hours yielded the highest polyphenol content. However, it is

325 understood that conditions of sun drying is not controlled throughout the process and oven

326 drying is the alternative option. Oven drying uses thermal energy to remove moisture from the

327 samples rapidly and at the same time, preserves the phytochemicals. Grinding of samples into

328 smaller particle size increases surface contact between samples and extraction solvents

329 (Azwanida NN, 2015; Hegde et al. 2015). These findings supported the use of oven drying at

$33060^{\circ} \mathrm{C}$ following by grinding for pre-extraction method with $C$. sinensis peels in this study.In this

331 study, we further identified the different phenolic acids, flavonoids and organic acids content in

332 the various extracts. Phenolic compounds are secondary metabolites synthesized by plants for

333 protection against excessive ultraviolet radiation or pathogenic aggression (Beckman, 2000).

334 Their biological benefits especially antioxidant properties have been extensively studied and

335 described in the literature. Five phenolic acids were identified from $C$. sinensis peel extracts

336 including gallic acid, protocatechuic acid, 4-hydroxybenzoic acid, caffeic acid and ferulic acid.

337 Ferulic acid is the most abundant phenolic acid of $C$. sinensis peel in our study, in agreement

338 with the previous study by M'hiri et al. (2017). Ferulic acid has been reported with various

339 bioactivities including antioxidant, anti-diabetic, anti-tumor and cardio-protection (Kumar \&

340 Pruthi, 2014). Similar to ferulic acid, caffeic acid which was present as the second most abundant

341 phenolic acid in all the extracts, are hydroxycinnamic acid derivatives which were shown to

342 show concentration-dependent antioxidant effects. These compounds exhibited inhibition against 
343 induced lipid peroxidation in mouse liver microsomes and scavenging activity against a range of

344 radicals including nitric oxide, superoxide and 2,2'-azino-bis-3-ethylbenzthiazoline-6-sulfonic

345 acid radical (ABTS+) (Maurya \& Devasagayam, 2010). In addition, both ferulic acid and caffeic

346 acid are widely added as active ingredients in cosmetic product due to its anti-aging, anti-

347 hyaluronidase and UV absorption capacities (Kumar \& Pruthi, 2014; Taofiq et al., 2017).

348 Besides, our results show that $C$. sinensis peel extracts contain flavonoids including catechin, 349 epigallocatechin, vitexin, rutin, luteolin and apigenin. Addition of water to the methanol and 350 ethanol extraction appeared to enhance the concentration of most flavonoids such as catechin, 351 epillocatechin, vitexin and luteolin (Table 4). All extracts with exception of 100\% MEC and $352100 \%$ EEC contained catechin as the most abundant flavonoid. Catechin is found abundantly in 353 tea extracts and is well known for its multiple health benefits including anti-aging, anti-diabetic 354 and anti-cancer effects (Pandey \& Rizvi, 2009). Epigallocatechin and apigenin which were also 355 present in high abundance in the extracts were suggested to be able to reverse epigenetic changes 356 in disease prevention and regulate a number of biological processes (Li et al., 2016; Shankar et al., 2016; Zhou, Yang and Kong, 2017). On the other hand, rutin was claimed to demonstrate beneficial biological properties including antioxidant, anti-inflammatory and anticarcinogenic properties (Rawson, Ho and Li, 2014).

Apart from the polyphenols, the concentration of organic acids were also determined via HPLC method (Table 4). In comparison to the other phytochemicals, lactic acid, citric acid and L-malic acid were present in much higher concentration in all the extracts apart from 100\% MEC, 100\% EEC and 70\% EEC. These organic acids are commonly found in citrus food and carry major economy value as they have been widely used as acidulant, preservative, emulsifier, flavorant and buffering agents across many industries particularly in food, beverage, pharmaceutical, nutraceutical and cosmetic manufacturing (Cirimina et al., 2017). For example, ascorbic acid and citric acid are normally added to fruit beverages, as acidulant, to enrich the nutrient content and palatability of juices from orange, grapefruit and lemon (Scherer et.al., 2012). In addition, the presence of ascorbic acid, limonoids citric acid and flavonoids content of $C$. sinensis had been claimed to be useful for fermentation and for treating kidney stones in clinical application (Alok et al., 2014). As global supply of organic acid particularly citric acid has rose from less than 0.5 to more than 2 million tonnes for the last two decades, extracting organic acid from citrus 
373 industrial waste can serve as alternative source that supplying the market needs of natural

374 organic acid (Cirimina et al., 2017).

\section{Conclusions}

376 The antioxidant activity, total phenolic and flavonoid content of the $C$. sinensis peels were

377 evaluated. The $C$. sinensis peels showed high antioxidant activities, total phenolic and flavonoid

378 content. Conventional solvent extraction in our study has produced extracts with high antioxidant

379 activities and high phytochemicals content. In particular, extraction of $C$. sinensis peels with 70

380 wt.\% acetone/water solvent was found to be most effective in extracting organic acid (citric acid

381 and lactic acid) and phenolic acid (ferrulic acid and caffeic acid) of the $C$. sinensis peel extracts.

382 However, for scale up industrial production, more effective technology can be considered such

383 as MAE and supercritical $\mathrm{CO}_{2}$ extraction.

384 The rich phytochemical constituents including phenolic and flavonoid content appeared to

385 contribute to the antioxidant potential to the $C$. sinensis peel extracts. The bioactive

386 phytochemicals could therefore be exploited for various applications such as for extraction of

387 natural antioxidants, food additive and colourants in the food industry. Despite being agricultural

388 wastes produced in the food supply chain, the enormous availability of $C$. sinensis peels could be

389 benefited as value added products in line with green technology.

390

391

392

393

394

395

396

397

398

399

\section{References}

Alok, S., Jain, S.K., Verma, A., kumar, M., Mahor, A., Sabharwal, M. (2014) 'Herbal antioxidant in clinical practice: A review', Asian Pacific Journal of Tropical Biomedicine, 4(1), pp. 78-84. doi: 10.1016/S2221-1691(14)60213-6.

Azwanida, N.N. (2015) 'A review on the extraction methods use in medicinal plants, principle, strength and limitation', Medicinal \& Aromatic Plants, 04(03). doi: 10.4172/21670412.1000196.

Beckman, C. H. (2000) 'Phenolic-storing cells: keys to programmed cell death and periderm formation in wilt disease resistance and in general defence responses in plants?', Physiological and Molecular Plant Pathology, 57(3), pp. 101-110. doi: 10.1006/PMPP.2000.0287. 
400 Benzie, I. F. F., Strain, J. J. (1996) 'The Ferric Reducing Ability of Plasma (FRAP) as a measure 401 of “antioxidant power": The FRAP assay', Analytical Biochemistry, 239(1), pp. 70-76. doi:

402

403

404

405

406

407

408

409

410

411

412

413

414

415

416

417

418

419

420

421

422

423

424

425

426

10.1006/abio.1996.0292.

Blauer, R. (2016) Citrus: World Markets and Trade | USDA Foreign Agricultural Service, USDA Foreign Agricultural Service. Available at: http://www.fas.usda.gov/data/citrus-world-marketsand-trade (Accessed: 28 February 2016).

Boudhrioua, N. (2016) 'Comparison of the efficiency of different extraction methods on antioxidants of maltease orange peel', International Journal of Food and Nutritional Science, 3(2), pp. 1-13. doi: 10.15436/2377-0619.16.789.

Brand-Williams, W., Cuvelier, M. E., Berset, C. (1995) 'Use of a free radical method to evaluate antioxidant activity', LWT - Food Science and Technology, 28(1), pp. 25-30. doi: 10.1016/S0023-6438(95)80008-5.

Ciriminna, R., Meneguzzo, F., Delisi, R., Pagliaro, M. (2017) 'Cirtic acid: emerging applications of key biotechnology industrial product', Chemistry Central Journal, 11, pp 22. doi: $\underline{10.1186 / \mathrm{s} 13065-017-0251-\mathrm{y}}$

Craft, BD., Kerrihard, A. L., Amarowicz, R., Pegg, R. B. (2012) 'Phenol-based antioxidants and the in vitro methods used for their assessment', Comprehensive Reviews in Food Science and Food Safety, pp. 148-73. doi: 10.1111/j.1541-4337.2011.00173.x

Dai, J., Mumper, R. J. (2010) 'Plant phenolics: Extraction, analysis and their antioxidant and anticancer properties', Molecules, 15(10), pp. 7313-7352. doi: 10.3390/molecules15107313.

Foreign Agricultural Service/USDA (2017) 'Citrus: World markets and trade', p. 12. Available at: https://public.govdelivery.com/accounts/USDAFAS/subscriber/new.

Genovese, S., Fiorito, S., Locatelli, M., Carlucci, G., Epifano, F. (2014) 'Analysis of biologically active oxyprenylated ferulic acid derivatives in citrus fruits', Plant Foods for Human Nutrition, 69(3), pp. 255-260. doi: 10.1007/s11130-014-0427-8.

Genovese, S., Epifano, F. (2012) 'Recent developments in the pharmacological properties of 4'geranyloxyferulic acid, a colon cancer chemopreventive agent of natural origin', Current Drug 
427 Targets, 13(8), pp. 1083-8. Available at: http://www.ncbi.nlm.nih.gov/pubmed/22594476

428 (Accessed: 10 June 2017).

429 Ghasemi, K., Ghasemi, Y., Ebrahimzadeh, M. A. (2009) 'Antioxidant activity, phenol and 430 flavonoid contents of 13 citrus species peels and tissues', Pakistan Journal of Pharmaceutical 431 Sciences, 22(3), pp. 277-81.

432 Huang, D., Ou, B., Hampsch-Woodil, M., Flanagan, J.A. Prior, R.L. (2002) 'High-throughput 433 assay of oxygen radical absorbance capacity (ORAC) using a multichannel liquid handling 434 system coupled with a microplate fluorescence reader in 96-well format', Journal of Agricultural 435 and Food Chemistry, 50(16), pp. 4437-4444. doi: 10.1021/jf0201529.

436 Ko, M.-J., Kwon, H.-L., Chung, M.-S. (2016) 'Pilot-scale subcritical water extraction of 437 flavonoids from satsuma mandarin (Citrus unshiu Markovich) peel', Innovative Food Science \& 438 Emerging Technologies, 38, pp. 175-181. doi: 10.1016/J.IFSET.2016.10.008.

439 Kumar, N., Pruthi, V. (2014). Potential applications of feruli acid from natural sources', 440 Biotechnology Reports, 4, pp. 86-93. doi: 10.1016/j.btre.2014.09.002

441 Li, B. B., Smith, B., Hossain, M. M. (2006) 'Extraction of phenolics from citrus peels', 442 Separation and Purification Technology, 48(2), pp. 182-188. doi: 10.1016/j.seppur.2005.07.005.

443 Li, S., Lo, C.-Y., Ho, C.-T. (2006) 'Hydroxylated polymethoxyflavones and methylated 444 flavonoids in sweet orange (Citrus sinensis) peel', Journal of Agricultural and Food Chemistry, 445 54(12), pp. 4176-85. doi: 10.1021/jf060234n.

446 Li, W., Guo, Y., Zhang, C., Wu, R., Yang, A.Y., Gaspar, J., Kong, A.N. (2016) 'Dietary 447 phytochemicals and cancer chemoprevention: A perspective on oxidative stress, inflammation, 448 and epigenetics', Chemical Research in Toxicology, 29(12), pp. 2071-2095. doi:

$44910.1021 /$ acs.chemrestox.6b00413.

450 M'hiri, N. Ioannou, I., Ghoul, M., Mihoubi Boudhrioua, N. (2017) 'Phytochemical 451 characteristics of citrus peel and effect of conventional and nonconventional processing on 452 phenolic compounds: A review', Food Reviews International, 33(6), pp. 587-619. doi: $453 \quad 10.1080 / 87559129.2016 .1196489$. 
454 Mamma, D., Christakopoulos, P. (2014) 'Biotransformation of citrus by-products into value 455 added products', Waste and Biomass Valorization, 5(4), pp. 529-549. doi: 10.1007/s12649-013456 9250-y.

457 Maurya, D. K., Devasagayam, T. P. A. (2010) 'Antioxidant and prooxidant nature of 458 hydroxycinnamic acid derivatives ferulic and caffeic acids', Food and Chemical Toxicology, 459 48(12), pp. 3369-3373. doi: 10.1016/j.fct.2010.09.006.

460 Naczk, M., Shahidi, F. (2006) 'Phenolics in cereals, fruits and vegetables: Occurrence, extraction 461 and analysis', Journal of Pharmaceutical and Biomedical Analysis, 41(5), pp.1523-1542. doi: 462 10.1016/j.jpba.2006.04.002.

463 Nayak, B., Dahmoune, F., Moussi, K., Remini, H., Dairi, S., Aoun, O., Khodir, M. (2015)

464 'Comparison of microwave, ultrasound and accelerated-assisted solvent extraction for recovery 465 of polyphenols from Citrus sinensis peels', Food Chemistry, 187, pp. 507-516. doi: 466 10.1016/j.foodchem.2015.04.081.

467 Onda, K., Horike, N., Suzuki, T., Hirano, T. (2013) 'Polymethoxyflavonoids tangeretin and 468 nobiletin increase glucose uptake in murine adipocytes', Phytotherapy Research, 27(2), pp. 312469 316. doi: 10.1002/ptr.4730.

470 Pandey, K. B., Rizvi, S. I. (2009) 'Plant polyphenols as dietary antioxidants in human health and 471 disease', Oxidative Medicine and Cellular Longevity, 2 (5), pp. 270-278, 472 doi:10.4161/oxim.2.5.9498

473 Prior, R. L., Wu, X., Schaich, K. (2005) 'Standardized methods for the determination of 474 antioxidant capacity and phenolics in foods and dietary supplements', Journal of Agricultural 475 and Food Chemistry, 53(10), pp. 4290-302. doi: 10.1021/jf0502698.

476 Qiu, P., Guan, H., Dong, P., Guo, S., Zheng, J., Li, S., Chen, Y., Ho, C.T., Pan, M.H., 477 McClements, D.J., Xiao, H. (2011) 'The inhibitory effects of 5-hydroxy-3,6,7,8,3',4'478 hexamethoxyflavone on human colon cancer cells', Molecular Nutrition \& Food Research, 479 55(10), pp. 1523-1532. doi: 10.1002/mnfr.201100070. 
480 Rafiq, S., Kaul, R., Sofi, S.A., Bashir, N., Nazir, F., Nayik, G.A. (2016) 'Citrus peel as a source 481 of functional ingredient: A review', Journal of the Saudi Society of Agricultural Sciences, in 482 press. doi: 10.1016/j.jssas.2016.07.006.

483 Rawson, N. E., Ho, C.-T., Li, S. (2014) 'Efficacious anti-cancer property of flavonoids from 484 citrus peels', Food Science and Human Wellness, 3(3-4), pp. 104-109. doi: 485 10.1016/j.fshw.2014.11.001.

486 Scherer, R. Rybka, A. C. P., Ballus, C. A., Meinhart, A. D., Filho, J. T., Godoy, H. T. (2012) 487 'Validation of a HPLC method for simultaneous determination of main organic acids in fruits 488 and juices', Food Chemistry, 135(1), pp 150-154. doi: 10.1016/j.foodchem.2012.03.111.

489 Selvamuthukumaran, M., Shi, J. (2017) 'Recent advances in extraction of antioxidants from 490 plant by-products processing industries', Food Quality and Safety, 1(1), pp. 61-81. doi:

$491 \quad 10.1093 /$ fqsafe/fyx004.

492 Shankar, E., Kanwal, R., Candamo, M., Gupta, S. (2016) 'Dietary phytochemicals as epigenetic 493 modifiers in cancer: Promise and challenges', Seminars in Cancer Biology. Academic Press, 40494 41, pp. 82-99. doi: 10.1016/J.SEMCANCER.2016.04.002.

495 Singleton, V. L. and Rossi, Joseph A. J. (1965) 'Colorimetry of total phenolics with 496 phosphomolybdic-phosphotungstic acid reagents', American Journal of Enology and Viticulture, 497 16(3), pp. 144-158.

498 Sousa, A., Ferreira, I.C.F.R., Barros, L., Bento, A., Pereira, J.A. (2008) 'Effect of solvent and 499 extraction temperatures on the antioxidant potential of traditional stoned table olives 500 “alcaparras", LWT - Food Science and Technology, 41(4), pp. 739-745. doi:

501 10.1016/J.LWT.2007.04.003.

502 Spigno, G., Tramelli, L., De Faveri, D.M. (2007). 'Effects of extraction time, temperature and 503 solvent on concentration and antioxidant activity of grape marc phenolics', Journal of Food 504 Engineering, 81(1), pp.200-208. doi: 10.1016/j.jfoodeng.2006.10.021.

505 Taofiq, O., Gonzalez-Paramas, A.M., Barreiro, M.F., Ferreira, I.C. (2017) 'Hydroxycinnamic 506 acids and their derivatives: Cosmeceutical significance, challenges and future perspectives, a 507 review', Molecules, 22(2), pp E281. doi: 10.3390/molecules22020281. 
508 Wong, C.-C., Li, H.B., Cheng, K.W., Chen, F. (2006) 'A systematic survey of antioxidant 509 activity of 30 Chinese medicinal plants using the ferric reducing antioxidant power assay', Food 510 Chemistry, 97(4), pp. 705-711. doi: 10.1016/J.FOODCHEM.2005.05.049

511 Zhishen, J., Mengcheng, T., Jianming, W. (1999) 'The determination of flavonoid contents in 512 mulberry and their scavenging effects on superoxide radicals', Food Chemistry, 64(4), pp. 555513 559. doi: 10.1016/S0308-8146(98)00102-2.

514 Zhou, Z., Yang, J., Kong, A.-N. (2017) 'Phytochemicals in traditional Chinese herbal medicine: 515 Cancer prevention and epigenetics mechanisms', Current Pharmacology Reports, 3(2), pp. 77516 91. doi: 10.1007/s40495-017-0086-1. 


\section{Table $\mathbf{1}$ (on next page)}

Antioxidant activities of $C$. sinensis peel extracts

abcdefgh Mean \pm SEM followed by different alphabets in the same column were significantly different between the $C$. sinensis peel extracts at $P<0.05$ by one-way ANOVA. TE, trolox equivalents; $\mathrm{Fe}(\mathrm{II})$, amount of $\mathrm{Fe}^{2+}$ reduced from $\mathrm{Fe}^{2+}$. Extracts of $C$. sinensis peel by using 100, 70, $50 \mathrm{wt}$ \% methanol/water and ethanol/water were expressed as $100 \% \mathrm{MEC}, 70 \%$ MEC, 50\% MEC, 100\% EEC, 70\% EEC, and 50\% EEC, respectively. Extracts of $C$. sinensis peel by using 70 and $50 \mathrm{wt} . \%$ acetone/water solvents were expressed as $70 \%$ AEC, and $50 \%$ AEC respectively. Water extract of $C$. sinensis peel were expressed as WEC. 
1 Table 1. Antioxidant activities of $C$. sinensis peel extracts.

\begin{tabular}{|c|c|c|c|}
\hline \multirow{3}{*}{ Sample } & DPPH & FRAP & ORAC \\
\hline & & & \\
\hline & (mg TE/g) & (mmol Fe(II)/g) & (mol TE/g) \\
\hline $100 \%$ MEC & $13.96 \pm 1.08^{a b c}$ & $275.62 \pm 1.85^{\mathrm{ab}}$ & $0.73 \pm 0.05^{\mathrm{a}}$ \\
\hline 70\% MEC & $16.69 \pm 1.20^{\mathrm{ab}}$ & $240.94 \pm 4.95^{c}$ & $0.70 \pm 0.04^{\mathrm{ab}}$ \\
\hline $50 \%$ MEC & $15.98 \pm 1.33^{\mathrm{ab}}$ & $214.64 \pm 3.49^{d}$ & $0.56 \pm 0.03^{\text {bcde }}$ \\
\hline $100 \%$ EEC & $11.61 \pm 0.82^{\mathrm{ac}}$ & $139.94 \pm 3.89^{e}$ & $0.46 \pm 0.03^{\text {cef }}$ \\
\hline $70 \%$ EEC & $16.52 \pm 1.29^{a b}$ & $219.02 \pm 3.87^{c d}$ & $0.68 \pm 0.02^{\mathrm{ad}}$ \\
\hline $50 \%$ EEC & $15.96 \pm 1.32^{\mathrm{ab}}$ & $194.73 \pm 5.81^{d}$ & $0.50 \pm 0.02^{\mathrm{cg}}$ \\
\hline $70 \%$ AEC & $18.20 \pm 1.62^{b}$ & $296.61 \pm 7.97^{a}$ & $0.92 \pm 0.03^{h}$ \\
\hline $50 \%$ AEC & $16.87 \pm 1.30^{\mathrm{ab}}$ & $269.71 \pm 7.33^{b}$ & $0.60 \pm 0.02^{\text {aeg }}$ \\
\hline WEC & $8.35 \pm 1.14^{c}$ & $95.00 \pm 2.11^{f}$ & $0.31 \pm 0.03^{f}$ \\
\hline Ascorbic acid & $1883.97 \pm 22.09$ & $14672.83 \pm 218.86$ & $5.33 \pm 0.69$ \\
\hline Gallic acid & $4133.73 \pm 360.07$ & $26059.73 \pm 4427.54$ & $7.88 \pm 0.60$ \\
\hline
\end{tabular}

abcdefgh Mean $\pm S E M$ followed by different alphabets in the same column were significantly different between the $C$. sinensis peel extracts at $\mathrm{P}<0.05$ by one-way ANOVA. TE, trolox equivalents; $\mathrm{Fe}(\mathrm{II})$, amount of $\mathrm{Fe}^{2+}$ reduced from $\mathrm{Fe}^{2+}$. Extracts of $C$. sinensis peel by using 100, 70, $50 \mathrm{wt} . \%$ methanol/water and ethanol/water were expressed as $100 \%$ $\mathrm{MEC}, 70 \% \mathrm{MEC}, 50 \% \mathrm{MEC}, 100 \% \mathrm{EEC}, 70 \% \mathrm{EEC}$, and $50 \% \mathrm{EEC}$, respectively. Extracts of $C$. sinensis peel by using 70 and 50 wt.\% acetone/water solvents were expressed as $70 \% \mathrm{AEC}$, and $50 \% \mathrm{AEC}$ respectively. Water extract of $C$. sinensis peel were expressed as WEC. 


\section{Table 2 (on next page)}

Correlation between antioxidant activities and phytoconstituents of $C$. sinensis peel extracts

${ }^{\text {a }}$ Correlation of the experimental values between the tests were statistical significant at $\mathrm{P}<$ 0.05 . 
1 Table 2. Correlation between antioxidant activities and phytoconstituents of $C$. sinensis peel extracts.

\begin{tabular}{|l|l|l|l|l|}
\hline Correlation R-square & DPPH & FRAP & ORAC & TFC \\
\hline TPC & $0.83^{\mathrm{a}}$ & $0.95^{\mathrm{a}}$ & $0.80^{\mathrm{a}}$ & $0.91^{\mathrm{a}}$ \\
\hline TFC & $0.76^{\mathrm{a}}$ & $0.93^{\mathrm{a}}$ & $0.66^{\mathrm{a}}$ & \\
\hline ORAC & $0.61^{\mathrm{a}}$ & $0.82^{\mathrm{a}}$ & & \\
\hline FRAP & $0.72^{\mathrm{a}}$ & & & \\
\hline
\end{tabular}

2 a Correlation of the experimental values between the tests were statistical significant at $\mathrm{P}<0.05$.

3

4

5 


\section{Table 3 (on next page)}

Extraction yield, total phenolic and flavonoid content of $C$. sinensis peel extracts abcdef Mean \pm SEM followed by different alphabets in the same column were significantly different at $\mathrm{P}<0.05$ by one-way ANOVA. DW, dry weight; GAE, gallic acid equivalents; $\mathrm{CE}$, catechin equivalents. Extracts of $C$. sinensis peel by using 100, 70, 50 wt.\% methanol/water and ethanol/water were expressed as 100\% MEC, $70 \%$ MEC, $50 \%$ MEC, $100 \%$ EEC, $70 \%$ EEC, and $50 \% \mathrm{EEC}$, respectively. Extracts of $C$. sinensis peel by using 70 and 50 wt.\% acetone/water solvents were expressed as 70\% AEC, and 50\% AEC respectively. Water extract of $C$. sinensis peel were expressed as WEC. 
1 Table 3. Extraction yield, total phenolic and flavonoid content of $C$. sinensis peel extracts.

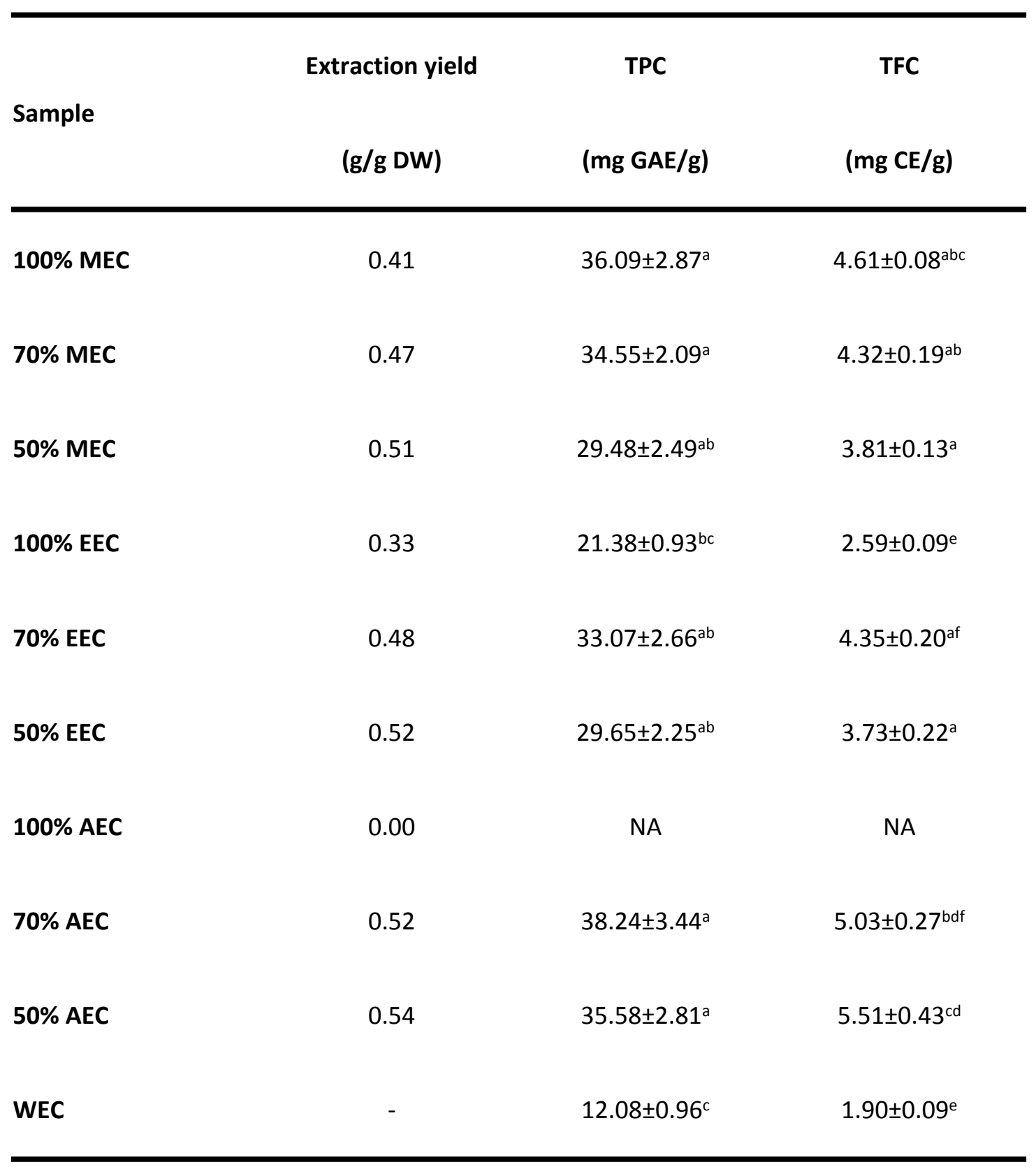

abcdef Mean \pm SEM followed by different alphabets in the same column were significantly different at $\mathrm{P}<0.05$ by one-way ANOVA. DW, dry weight; GAE, gallic acid equivalents; $C E$, catechin equivalents. Extracts of $C$. sinensis peel 5 by using 100, 70, $50 \mathrm{wt} . \%$ methanol/water and ethanol/water were expressed as $100 \% \mathrm{MEC}, 70 \% \mathrm{MEC}, 50 \% \mathrm{MEC}$, $6100 \% \mathrm{EEC}, 70 \% \mathrm{EEC}$, and $50 \% \mathrm{EEC}$, respectively. Extracts of $C$. sinensis peel using 100, 70 and $50 \mathrm{wt} . \%$

7 acetone/water solvents were expressed as $100 \% \mathrm{AEC}, 70 \% \mathrm{AEC}$, and $50 \% \mathrm{AEC}$ respectively. Water extract of $C$.

8 sinensis peel were expressed as WEC. NA: Not available (The yield of $100 \%$ acetone was lower than $0.005 \mathrm{~g} / \mathrm{g} \mathrm{DW}$

9 and was not soluble, thus not able to be used for subsequent analysis.) 


\section{Table 4 (on next page)}

Phytochemical content of $C$. sinensis peel extracts.

Each data point indicates the average results of phytochemicals phenolic acid, organic acid and flavonoids in $\mathrm{ug} / \mathrm{g}$ extract of $\mathrm{C}$ sinensis peel extracts. 
Table 4. Phytochemical content of $C$. sinensis peel extracts.

\begin{tabular}{|c|c|c|c|c|c|c|c|c|c|}
\hline $\begin{array}{l}\text { Phytochemicals } \\
\text { (ug/g extract) }\end{array}$ & $100 \% \mathrm{MEC}$ & $70 \%$ MEC & $50 \%$ MEC & $100 \%$ EEC & $70 \%$ EEC & $50 \%$ EEC & $70 \%$ AEC & $50 \%$ AEC & WEC \\
\hline \multicolumn{10}{|l|}{ Phenolic acid } \\
\hline Gallic acid & 43.43 & 18.80 & 32.69 & 31.30 & 42.67 & 38.88 & 33.55 & 40.14 & 20.14 \\
\hline Protocatechuic acid & 59.87 & 140.25 & 108.95 & 70.33 & 121.25 & 133.26 & 112.08 & 130.79 & 24.40 \\
\hline 4-hydroxybenzoic acid & 65.22 & 53.93 & 69.79 & 63.48 & 73.05 & 81.74 & 50.51 & 54.25 & 24.07 \\
\hline Caffeic acid & 224.65 & 247.96 & 411.75 & 164.99 & 243.01 & 362.70 & 266.43 & 264.84 & 69.58 \\
\hline Ferulic acid & 377.61 & 821.87 & 769.19 & 579.33 & 404.34 & 742.22 & 917.88 & 683.44 & 108.79 \\
\hline \multicolumn{10}{|l|}{ Organic acid } \\
\hline Lactic acid & 18660.69 & 20981.33 & 16387.22 & 20190.94 & 16316.47 & 12708.52 & 12444.50 & 10117.39 & 20929.25 \\
\hline Citric acid & 0 & 40900.07 & 38405.91 & 0 & 0 & 42559.18 & 53673.85 & 45076.92 & 37364.21 \\
\hline L-mallic acid & 0 & 9207.74 & 9200.43 & 0 & 0 & 8701.07 & 5876.59 & 9378.31 & 1308.75 \\
\hline Kojic acid & 0 & 247.64 & 218.13 & 0 & 0 & 222.96 & 221.39 & 229.17 & 120.68 \\
\hline Ascorbic acid & 0 & 12.55 & 2.19 & 0 & 0 & 4.37 & 9.71 & 13.55 & 6.21 \\
\hline \multicolumn{10}{|l|}{ Flavonoids } \\
\hline Catechin & 248.76 & 514.63 & 627.22 & 123.99 & 573.81 & 669.62 & 572.26 & 679.32 & 352.72 \\
\hline Epigallocatechin & 373.41 & 472.66 & 621.84 & 255.24 & 436.16 & 593.99 & 446.57 & 520.82 & 178.07 \\
\hline Vitexin & 103.92 & 190.17 & 196.80 & 93.12 & 107.60 & 202.40 & 225.52 & 195.38 & 69.61 \\
\hline Rutin & 23.48 & 20.34 & 20.38 & 30.63 & 29.48 & 22.36 & 21.63 & 26.63 & 15.08 \\
\hline
\end{tabular}




\begin{tabular}{|c|c|c|c|c|c|c|c|c|c|}
\hline Luteolin & 227.97 & 496.83 & 539.49 & 334.51 & 300.51 & 458.37 & 516.27 & 478.70 & 324.74 \\
\hline Apigenin & 141.24 & 285.77 & 313.08 & 203.65 & 174.91 & 270.45 & 311.19 & 279.59 & 194.38 \\
\hline
\end{tabular}

4 Each data point indicates the average results of phytochemicals phenolic acid, organic acid and flavonoids in ug/g extract of $C$ sinensis peel extracts. 